PROCEEDINGS OF THE

AMERICAN MATHEMATICAL SOCIETY

Volume 136, Number 11, November 2008, Pages 3869-3874

S 0002-9939(08)09582-8

Article electronically published on June 24, 2008

\title{
A BANACH-STONE THEOREM FOR RIESZ ISOMORPHISMS OF BANACH LATTICES
}

\author{
JIN XI CHEN, ZI LI CHEN, AND NGAI-CHING WONG
}

(Communicated by N. Tomczak-Jaegermann)

\begin{abstract}
Let $X$ and $Y$ be compact Hausdorff spaces, and $E, F$ be Banach lattices. Let $C(X, E)$ denote the Banach lattice of all continuous $E$-valued functions on $X$ equipped with the pointwise ordering and the sup norm. We prove that if there exists a Riesz isomorphism $\Phi: C(X, E) \rightarrow C(Y, F)$ such that $\Phi f$ is non-vanishing on $Y$ if and only if $f$ is non-vanishing on $X$, then $X$ is homeomorphic to $Y$, and $E$ is Riesz isomorphic to $F$. In this case, $\Phi$ can be written as a weighted composition operator: $\Phi f(y)=\Pi(y)(f(\varphi(y)))$, where $\varphi$ is a homeomorphism from $Y$ onto $X$, and $\Pi(y)$ is a Riesz isomorphism from $E$ onto $F$ for every $y$ in $Y$. This generalizes some known results obtained recently.
\end{abstract}

\section{INTRODUCTION}

Let $X$ and $Y$ be compact Hausdorff spaces, and $C(X), C(Y)$ denote the spaces of real-valued continuous functions defined on $X, Y$ respectively. There are three versions of the Banach-Stone theorem. That is to say, surjective linear isometries, ring isomorphisms and lattice isomorphisms from $C(X)$ onto $C(Y)$ yield homeomorphisms between $X$ and $Y$, respectively (cf. 11, 6, 14).

Jerison [13] got the first vector-valued version of the Banach-Stone theorem. He proved that if the Banach space $E$ is strictly convex, then every surjective linear isometry $\Phi: C(X, E) \rightarrow C(Y, E)$ can be written as a weighted composition operator

$$
\Phi f(y)=\Pi(y)(f(\varphi(y))), \quad \forall f \in C(X, E), \forall y \in Y .
$$

Here $\varphi$ is a homeomorphism from $Y$ onto $X$, and $\Pi$ is a continuous map from $Y$ into the space $(\mathcal{L}(E, E), S O T)$ of bounded linear operators on $E$ equipped with the strong operator topology $(S O T)$. Furthermore, $\Pi(y)$ is a surjective linear isometry on $E$ for every $y$ in $Y$. After Jerison [13, many vector-valued versions of the Banach-Stone theorem have been obtained in different ways (see, e.g., 3, 4, 5, 7, 9, 10, 12, 16]).

Let $E, F$ be non-zero real Banach lattices, and $C(X, E)$ be the Banach lattice of all continuous $E$-valued functions on $X$ equipped with the pointwise ordering and the sup norm. Note that, in general, a Riesz isomorphism (i.e., lattice isomorphism ) from $C(X, E)$ onto $C(Y, F)$ does not necessarily induce a topological

Received by the editors June 1, 2007.

2000 Mathematics Subject Classification. Primary 46E40; Secondary 46B42, 47B65.

Key words and phrases. Banach lattice, Banach-Stone theorem, Riesz isomorphism, weighted composition operator.

(C)2008 American Mathematical Society Reverts to public domain 28 years from publication 
homeomorphism from $X$ onto $Y$ (cf. [16, Example 3.5]). To consider the BanachStone theorems for continuous Banach lattice-valued functions, we would like to mention the papers [5, 7, 16. In particular, when $E, F$ are both Banach lattices and Riesz algebras, Miao, Cao and Xiong [16] recently proved that if $F$ has no zerodivisor and there exists a Riesz algebraic isomorphism $\Phi: C(X, E) \rightarrow C(Y, F)$ such that $\Phi f$ is non-vanishing on $Y$ if $f$ is non-vanishing on $X$, then $X$ is homeomorphic to $Y$, and $E$ is Riesz algebraically isomorphic to $F$. By saying $f$ in $C(X, E)$ is nonvanishing, we mean that $0 \notin f(X)$. Indeed, under these conditions they obtained that $\Phi^{-1} g$ is non-vanishing on $X$ if $g \in C(Y, F)$ is non-vanishing on $Y$. Note that every Riesz algebraic isomorphism must be a Riesz isomorphism.

Let $E$ and $F$ be Banach lattices. More recently, Ercan and Önal $[7$ have established that if $F$ is an $A M$-space with unit, i.e., a $C(K)$-space, and there exists a Riesz isomorphism $\Phi: C(X, E) \rightarrow C(Y, F)$ such that $\Phi f$ is non-vanishing on $Y$ if and only if $f$ is non-vanishing on $X$, that is, both $\Phi$ and $\Phi^{-1}$ are non-vanishing preserving, then $X$ is homeomorphic to $Y$ and $E$ is Riesz isomorphic to $F$.

Inspired by [5, 7, 16, one can ask a natural question:

Question 1. Is $X$ homeomorphic to $Y$ if $E, F$ are Banach lattices and there exists a Riesz isomorphism $\Phi: C(X, E) \rightarrow C(Y, F)$ such that both $\Phi$ and $\Phi^{-1}$ are non-vanishing preserving?

In this paper we show the answer to the above question is affirmative. Moreover, in this case $\Phi$ can be written as a weighted composition operator:

$$
\Phi f(y)=\Pi(y)(f(\varphi(y))), \quad \forall f \in C(X, E), \forall y \in Y,
$$

where $\varphi$ is a homeomorphism from $Y$ onto $X$, and $\Pi(y)$ is a Riesz isomorphism from $E$ onto $F$ for every $y$ in $Y$. This generalizes the results obtained by Cao, Reilly and Xiong [5], Miao, Cao, and Xiong [16], and Ercan and Önal 7].

Our notions are standard. For the undefined notions and basic facts concerning Banach lattices we refer the reader to the monographs [1, 2, 14].

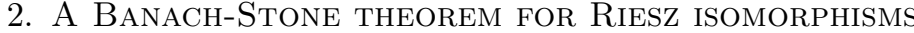

In the following we always assume $X$ and $Y$ are compact Hausdorff spaces, $E$ and $F$ are non-zero Banach lattices, and $\mathcal{L}(E, F)$ is the space of bounded linear operators from $E$ into $F$ equipped with $S O T$. For $x$ in $X$ and $y$ in $Y$, let $M_{x}$ and $N_{y}$ be defined as

$$
M_{x}=\{f \in C(X, E): f(x)=0\}, \quad N_{y}=\{g \in C(Y, F): g(y)=0\} .
$$

Clearly, $M_{x}$ and $N_{y}$ are closed (order) ideals in $C(X, E)$ and $C(Y, F)$, respectively.

Lemma 2. Let $\Phi: C(X, E) \rightarrow C(Y, F)$ be a Riesz isomorphism such that $\Phi(f)$ is non-vanishing on $Y$ if and only if $f$ is non-vanishing on $X$. Then for each $x$ in $X$ there exists a unique $y$ in $Y$ such that

$$
\Phi M_{x}=N_{y} .
$$

In particular, this defines a bijection $\varphi$ from $Y$ onto $X$ by $\varphi(y)=x$.

Proof. For each $x$ in $X$, let

$$
\mathcal{Z}\left(\Phi M_{x}\right)=\left\{y \in Y: \Phi f(y)=0 \text { for all } f \in M_{x}\right\} .
$$

We first claim that $\mathcal{Z}\left(\Phi M_{x}\right)$ is non-empty. Suppose, on the contrary, that $\mathcal{Z}\left(\Phi M_{x}\right)$ is empty. Then for each $y$ in $Y$ there would exist an $f_{y}$ in $M_{x}$ such that $\Phi f_{y}(y) \neq 0$, 
and thus $\Phi f_{y}$ is non-vanishing in an open neighborhood of $y$. Note that $\left|f_{y}\right| \in M_{x}$, and $\Phi\left|f_{y}\right|=\left|\Phi f_{y}\right|$ since $\Phi$ is a Riesz isomorphism. Therefore, we can assume further that both $f_{y}$ and $\Phi f_{y}$ are positive by replacing them by their absolute values if necessary. By the compactness of $Y$, we can choose finitely many $f_{1}, \ldots, f_{n}$ from $M_{x}^{+}$such that the positive functions $\Phi f_{1}, \ldots, \Phi f_{n}$ have no common zero in $Y$. Hence $\Phi\left(f_{1}+\cdots+f_{n}\right)$ is strictly positive; that is, $\Phi\left(f_{1}+\cdots+f_{n}\right)(y)>0$ for each $y$ in $Y$. This contradicts the fact that $f_{1}+\cdots+f_{n}$ vanishes at $x$. We thus prove that $\mathcal{Z}\left(\Phi M_{x}\right) \neq \phi$.

Next, we claim that $\mathcal{Z}\left(\Phi M_{x}\right)$ is a singleton. Indeed, if $y_{1}, y_{2} \in \mathcal{Z}\left(\Phi M_{x}\right)$, then we would have $\Phi M_{x} \subseteq N_{y_{i}}, i=1,2$. Applying the above argument to $\Phi^{-1}$, we shall have $\Phi^{-1} N_{y_{i}} \subseteq M_{x_{i}}$ for some $x_{i}$ in $X, i=1,2$. It follows that $\Phi M_{x} \subseteq N_{y_{i}} \subseteq$ $\Phi M_{x_{i}}, i=1,2$. Then $x=x_{1}=x_{2}$ since $\Phi$ is bijective and $X$ is Hausdorff. Thus,

$$
y_{1}=y_{2} \quad \text { and } \quad \Phi M_{x}=N_{y_{1}}=N_{y_{2}}
$$

Now, we can define a bijective map $\varphi: Y \rightarrow X$ such that

$$
\Phi M_{\varphi(y)}=N_{y}, \quad \forall y \in Y
$$

The following main result answers affirmatively the question mentioned in the introduction and solves the conjecture of Ercan and Önal in [7].

Theorem 3. Let $\Phi: C(X, E) \rightarrow C(Y, F)$ be a Riesz isomorphism such that $\Phi f$ is non-vanishing on $Y$ if and only if $f$ is non-vanishing on $X$. Then $Y$ is homeomorphic to $X$, and $\Phi$ can be written as a weighted composition operator

$$
\Phi f(y)=\Pi(y)(f(\varphi(y))), \quad \forall f \in C(X, E), \forall y \in Y
$$

Here $\varphi$ is a homeomorphism from $Y$ onto $X$, and $\Pi(y)$ is a Riesz isomorphism from $E$ onto $F$ for every $y$ in $Y$. Moreover, $\Pi: Y \rightarrow(\mathcal{L}(E, F), S O T)$ is continuous, and $\|\Phi\|=\sup _{y \in Y}\|\Pi(y)\|$.

Proof. First, we show that the bijection $\varphi$ given in Lemma 2 is a homeomorphism from $Y$ onto $X$. It suffices to verify the continuity of $\varphi$ since $Y$ is compact and $X$ is Hausdorff. To this end, suppose, to the contrary, that there would exist a net $\left\{y_{\lambda}\right\}$ in $Y$ converging to $y_{0}$ in $Y$, but $\varphi\left(y_{\lambda}\right)$ converges to $x_{0} \neq \varphi\left(y_{0}\right)$ in $X$.

Let $U_{x_{0}}$ and $U_{\varphi\left(y_{0}\right)}$ be disjoint open neighborhoods of $x_{0}$ and $\varphi\left(y_{0}\right)$, respectively. First, for any $f$ in $C(X, E)$ vanishing outside $U_{\varphi\left(y_{0}\right)}$ we claim that $\Phi f\left(y_{0}\right)=0$. Indeed, since $\varphi\left(y_{\lambda}\right)$ belongs to $U_{x_{0}}$ for $\lambda$ large enough and $f(x)=0$ for any $x$ in $U_{x_{0}}$, we have that $f \in M_{\varphi\left(y_{\lambda}\right)}$. It follows from Lemma 2 that $\Phi f \in N_{y_{\lambda}}$; that is, $\Phi f\left(y_{\lambda}\right)=0$ when $\lambda$ is large enough. Thus, $\Phi f\left(y_{0}\right)=0$ since $y_{\lambda} \rightarrow y_{0}$ and $\Phi f$ is continuous.

Let $\chi \in C(X)$ such that $\chi$ vanishes outside $U_{\varphi\left(y_{0}\right)}$ and $\chi\left(\varphi\left(y_{0}\right)\right)=1$. Then, for any $h$ in $C(X, E)$, we have $h=\chi h+(1-\chi) h$. Since $\chi h$ vanishes outside $U_{\varphi\left(y_{0}\right)}$, by the above argument, we can see that $\Phi(\chi h)\left(y_{0}\right)=0$. Clearly, $\Phi((1-\chi) h)$ vanishes at $y_{0}$ since $(1-\chi) h \in M_{\varphi\left(y_{0}\right)}$. Thus, $\Phi h\left(y_{0}\right)=\Phi(\chi h)\left(y_{0}\right)+\Phi((1-\chi) h)\left(y_{0}\right)=0$ for any $h$ in $C(X, E)$. This leads to a contradiction since $\Phi$ is surjective. So $\varphi$ is continuous and thus a homeomorphism from $Y$ onto $X$ satisfying $\Phi M_{\varphi(y)}=N_{y}$ for each $y$ in $Y$. 
Next, note that $\operatorname{ker} \delta_{\varphi(y)}=\operatorname{ker} \delta_{y} \circ \Phi$, where $\delta_{y}$ is the Dirac functional. Hence, there is a linear operator $\Pi(y): E \rightarrow F$ such that $\delta_{y} \circ \Phi=\Pi(y) \circ \delta_{\varphi(y)}$. In other words,

$$
\Phi f(y)=\Pi(y)(f(\varphi(y))), \quad \forall f \in C(X, E), \forall y \in Y .
$$

See, e.g., 8, p. 67].

It is routine to verify the other assertions in the statement of this theorem. For the convenience of the reader, we give a sketch of the rest of the proof. For $e$ in $E$, let $\mathbf{1}_{X} \otimes e \in C(X, E)$ be defined by $\left(\mathbf{1}_{X} \otimes e\right)(x)=e$ for each $x$ in $X$. Let $y$ in $Y$ be fixed. If $e \neq 0$, then $\Pi(y) e=\Pi(y)\left(\left(\mathbf{1}_{X} \otimes e\right)(\varphi(y))\right)=\Phi\left(\mathbf{1}_{X} \otimes e\right)(y) \neq 0$ since $\mathbf{1}_{X} \otimes e$ is non-vanishing. Hence, $\Pi(y)$ is one-to-one. On the other hand, for $u$ in $F$ we can find a function $f$ in $C(X, E)$ such that $\Phi f=\mathbf{1}_{Y} \otimes u$ by the surjectivity of $\Phi$. Let $e=f(\varphi(y))$. Then $\Pi(y) e=\Pi(y)(f(\varphi(y)))=\Phi f(y)=u$. That is, $\Pi(y)$ is surjective. To see that $\Pi(y)$ is a Riesz isomorphism, let $e_{1}, e_{2} \in E$. Then $\Pi(y)\left(e_{1} \vee e_{2}\right)=\Phi\left(\mathbf{1}_{X} \otimes\left(e_{1} \vee e_{2}\right)\right)(y)=\Phi\left(\mathbf{1}_{X} \otimes e_{1}\right)(y) \vee \Phi\left(\mathbf{1}_{X} \otimes e_{2}\right)(y)=$ $\Pi(y) e_{1} \vee \Pi(y) e_{2}$, since $\Phi$ is a Riesz isomorphism.

Recall that every positive operator between Banach lattices is continuous. Let $e \in E$. Since $\|\Pi(y) e\|=\left\|\Phi\left(\mathbf{1}_{X} \otimes e\right)(y)\right\| \leq\left\|\Phi\left(\mathbf{1}_{X} \otimes e\right)\right\| \leq\|\Phi\|\|e\|$, we have $\|\Pi(y)\| \leq\|\Phi\|$ for all $y$ in $Y$. On the other hand, for any $f$ in $C(X, E)$ and any $y$ in $Y$, we can see that $\|\Phi f(y)\|=\|\Pi(y)(f(\varphi(y)))\| \leq\|\Pi(y)\|\|f\|$. Consequently, $\|\Phi\| \leq \sup _{y \in Y}\|\Pi(y)\|$.

Finally, we prove that $\Pi: Y \rightarrow(\mathcal{L}(E, F), S O T)$ is continuous. To this end, let $\left\{y_{\lambda}\right\}$ be a net such that $y_{\lambda} \rightarrow y$ in $Y$. Then, for any $e$ in $E,\left\|\Pi\left(y_{\lambda}\right) e-\Pi(y) e\right\|=$ $\left\|\Phi\left(\mathbf{1}_{X} \otimes e\right)\left(y_{\lambda}\right)-\Phi\left(\mathbf{1}_{X} \otimes e\right)(y)\right\| \rightarrow 0$, since $\Phi\left(\mathbf{1}_{X} \otimes e\right)$ is continuous on $Y$.

In the above results, we have to assume that both $\Phi$ and $\Phi^{-1}$ are non-vanishing preserving. In the following example, we can see that the inverse of a non-vanishing preserving Riesz isomorphism is not necessarily non-vanishing preserving.

Example 4. Let $X=\{1,2\}$ be equipped with the discrete topology, let $E=\mathbb{R}$ have its usual ordering and norm, and let $Y=\{0\}$ and $F=\mathbb{R}^{2}$ with the pointwise ordering and the sup norm. Define $\Phi: C(X, E) \rightarrow C(Y, F)$ by $\Phi f(0)=(f(1), f(2))$. Clearly, the Riesz isometric isomorphism $\Phi$ is non-vanishing preserving, but its inverse $\Phi^{-1}$ is not.

Let $E, F$ be both Banach lattices and Riesz algebras. Miao, Cao and Xiong [16] recently proved that if $F$ has no zero-divisor and there exists a Riesz algebraic isomorphism $\Phi: C(X, E) \rightarrow C(Y, F)$ such that $\Phi f$ is non-vanishing on $Y$ if $f$ is non-vanishing on $X$, then $X$ is homeomorphic to $Y$ and $E$ is Riesz algebraically isomorphic to $F$. In fact, from their proof we can see that $\Phi f$ is non-vanishing on $Y$ if and only if $f$ is non-vanishing on $X$; that is, both $\Phi$ and $\Phi^{-1}$ are non-vanishing preserving. Therefore, the result of Miao, Cao and Xiong can be restated as follows.

Corollary 5 (16]). Let $E, F$ be both Banach lattices and Riesz algebras. If $F$ has no zero-divisor and $\Phi: C(X, E) \rightarrow C(Y, F)$ is a Riesz algebraic isomorphism such that $\Phi f$ is non-vanishing on $Y$ if $f$ is non-vanishing on $X$, then $\Phi$ is a weighted composition operator

$$
\Phi f(y)=\Pi(y)(f(\varphi(y))), \quad \forall f \in C(X, E), \forall y \in Y .
$$

Here $\varphi$ is a homeomorphism from $Y$ onto $X$, and $\Pi(y)$ is a Riesz algebraic isomorphism from $E$ onto $F$ for every $y$ in $Y$. 
In Theorem 3, when $X, Y$ are compact Hausdorff spaces and $E=F=\mathbb{R}$, the lattice hypothesis about $\Phi$ can be dropped.

Example 6. Let $X, Y$ be compact Hausdorff spaces, and $C(X), C(Y)$ be the Banach spaces of continuous real-valued functions defined on $X, Y$, respectively. Assume $\Phi: C(X) \rightarrow C(Y)$ is a linear map such that $\Phi f$ is non-vanishing on $Y$ if and only if $f$ is non-vanishing on $X$.

Note that $\left(\Phi \mathbf{1}_{X}\right)^{-1} \Phi$ is a unital linear map preserving non-vanishing. Let $\lambda$ be in the range of $f$. Then $f-\lambda \mathbf{1}_{X}$ is not invertible, and thus neither is $\left(\Phi \mathbf{1}_{X}\right)^{-1} \Phi f-\lambda \mathbf{1}_{Y}$. It follows that $\lambda$ is in the range of $\left(\Phi \mathbf{1}_{X}\right)^{-1} \Phi f$. The converse also holds. Therefore, the range of $\left(\Phi \mathbf{1}_{X}\right)^{-1} \Phi f$ coincides with the range of $f$ for each $f$ in $C(X)$. In particular, $\left(\Phi \mathbf{1}_{X}\right)^{-1} \Phi$ is a unital linear isometry from $C(X)$ into $C(Y)$. By the Holsztyński Theorem [11, there is a compact subset $Y_{0}$ of $Y$ and a quotient map $\varphi: Y_{0} \rightarrow X$ such that

$$
\left.\left(\Phi \mathbf{1}_{X}\right)^{-1} \Phi f\right|_{Y_{0}}=f \circ \varphi, \quad \forall f \in C(X) .
$$

In case $\Phi$ is surjective, the classical Banach-Stone Theorem ensures that $\varphi$ is a homeomorphism from $Y=Y_{0}$ onto $X$. Moreover, if $\Phi \mathbf{1}_{X}$ is strictly positive on $Y$, then $\Phi$ is a Riesz isomorphism. However, when $\Phi$ is not surjective the situation is a bit uncontrollable. For example, consider $\Phi: C[0,1] \rightarrow C\left(\left[0, \frac{1}{2}\right] \cup\left[1, \frac{3}{2}\right]\right)$ defined by

$$
\Phi f(y)= \begin{cases}f(2 y), & \text { if } 0 \leq y \leq 1 / 2 \\ (2 y-2) f(0)+(3-2 y) f(1), & \text { if } 1 \leq y \leq \frac{3}{2}\end{cases}
$$

Clearly, the thus defined $\Phi$ is a non-surjective linear isometry preserving nonvanishing in two ways, but $[0,1]$ is not homeomorphic to $\left[0, \frac{1}{2}\right] \cup\left[1, \frac{3}{2}\right]$.

Finally, we borrow an example from [15] which shows that the surjectivity cannot be guaranteed by many other properties we usually consider.

Example 7. Let $\omega$ and $\omega_{1}$ be the first infinite and the first uncountable ordinal numbers, respectively. Let $\left[0, \omega_{1}\right]$ be the compact Hausdorff space consisting of all ordinal numbers $x$ not greater than $\omega_{1}$ and equipped with the topology generated by order intervals. Note that every continuous function $f$ in $C\left[0, \omega_{1}\right]$ is eventually constant. More precisely, there is a non-limit ordinal $x_{f}$ such that $\omega<x_{f}<\omega_{1}$ and $f(x)=f\left(\omega_{1}\right)$ for all $x \geq x_{f}$.

Define $\phi:\left[0, \omega_{1}\right] \rightarrow\left[0, \omega_{1}\right]$ by setting

$$
\phi(0)=\omega_{1}, \quad \phi(n)=n-1 \text { for all } n=1,2, \ldots, \quad \text { and } \phi(x)=x \text { for all } x \geq \omega .
$$

Let $\Phi: C\left[0, \omega_{1}\right] \rightarrow C\left[0, \omega_{1}\right]$ be the non-surjective composition operator defined by $\Phi f=f \circ \phi$. It is plain that $\Phi$ is an isometric unital algebraic and lattice isomorphism from $C\left[0, \omega_{1}\right]$ onto its range. In fact, one can see in [15, Example 3.3] that the map $\Phi$ is a non-surjective linear $n$-local automorphism of $C\left[0, \omega_{1}\right]$, where $n=1,2, \ldots, \omega$; i.e., the action of $\Phi$ on any set $S$ of cardinality not greater than $n$ agrees with an automorphism $\Phi_{S}$.

\section{ACKNOWLEDGMENT}

The authors would like to thank the referee for comments which have improved this paper. 


\section{REFERENCES}

[1] Y.A. Abramovich and C.D. Aliprantis, An Invitation to Operator Theory, Graduate Studies in Mathematics 50, American Mathematical Society, Providence, RI, 2002. MR2003h:47072

[2] C.D. Aliprantis and O. Burkinshaw, Positive Operators, Pure and Applied Mathematics 119, Academic Press, New York, 1985. MR 87h:47086

[3] E. Behrends, How to obtain vector-valued Banach-Stone theorems by using $M$-structure methods, Math. Ann. 261 (1982), 387-398. MR84c:46012

[4] E. Behrends and M. Cambern, An isomorphic Banach-Stone theorem, Studia Math. 90 (1988), 15-26. MR:89h:46020

[5] J. Cao, I. Reilly and H. Xiong, A lattice-valued Banach-Stone theorem, Acta Math. Hungar. 98 (2003), 103-110. MR2003m:46028

[6] J.B. Conway, A Course in Functional Analysis, Second edition, Graduate Texts in Mathematics 96, Springer, New York, 1990. MR91e:46001

[7] Z. Ercan and S. Önal, Banach-Stone theorem for Banach lattice valued continuous functions, Proc. Amer. Math. Soc. 135 (2007), 2827-2829. MR2008a:46038

[8] M. Fabian et al., Functional Analysis and Infinite-Dimensional Geometry, CMS Books in Mathematics 8, Springer, New York, 2001. MR2002f:46001

[9] H.-L. Gau, J.-S. Jeang and N.-C. Wong, Biseparating linear maps between continuous vectorvalued function spaces, J. Aust. Math. Soc. 74 (2003), 101-109. MR2003j:47041

[10] S. Hernandez, E. Beckenstein and L. Narici, Banach-Stone theorems and separating maps, Manuscr. Math. 86 (1995), 409-416. MR95m:46054

[11] W. Holsztyński, Continuous mappings induced by isometries of spaces of continuous function, Studia Math. 26 (1966), 133-136. MR 33:1711

[12] J.-S. Jeang and N.-C. Wong, On the Banach-Stone problem, Studia Math. 155 (2003), 95105. MR2004a:46039

[13] M. Jerison, The space of bounded maps into a Banach space, Ann. of Math.(2) 52 (1950), 309-327. MR 12:188c

[14] E. de Jonge and A. van Rooij, Introduction to Riesz Spaces, Mathematical Centre Tracts 78, Amsterdam, 1977. MR.57:13439

[15] J.-H. Liu and N.-C. Wong, Local automorphisms of operator algebras, Taiwanese J. Math. 11 (2007), 611-619. MR.2340153

[16] X. Miao, J. Cao and H. Xiong, Banach-Stone theorems and Riesz algebras, J. Math. Anal. Appl. 313 (2006), 177-183. MR2006m:46030

Department of Mathematics, Southwest Jiaotong University, Chengdu 610031, People's Republic of China

E-mail address: jinxichen@home.swjtu.edu.cn

Department of Mathematics, Southwest Jiaotong University, Chengdu 610031, People's Republic of China

E-mail address: zlchen@home.swjtu.edu.cn

Department of Applied Mathematics, National Sun Yat-Sen University, Kaohsiung 80424, TAIWAN

E-mail address: wong@math.nsysu.edu.tw 